\title{
Relationship between domain-boundary free energy and the temperature dependence of stress-domain patterns of $\mathrm{Pb}$ on $\mathrm{Cu}(111)$
}

\author{
R. van Gastel, ${ }^{1,2}$ N. C. Bartelt, ${ }^{3}$ P. J. Feibelman, ${ }^{1}$ F. Léonard, ${ }^{3}$ and G. L. Kellogg ${ }^{1}$ \\ ${ }^{1}$ Sandia National Laboratories, Albuquerque, New Mexico 87185-1415, USA \\ ${ }^{2}$ Solid State Physics, MESA + Institute, University of Twente, 7500 AE Enschede, The Netherlands \\ ${ }^{3}$ Sandia National Laboratories, Livermore, California 94551-0969, USA \\ (Received 23 June 2004; revised manuscript received 19 August 2004; published 9 December 2004)
}

\begin{abstract}
$\mathrm{Pb}$ deposition on $\mathrm{Cu}(111)$ causes the surface to self-assemble into periodically arranged domains of a $\mathrm{Pb}$-rich phase and a $\mathrm{Pb}$-poor phase. Using low-energy electron microscopy (LEEM) we provide evidence that the observed temperature-dependent periodicity of these self-assembled domain patterns is the result of changing domain-boundary free energy. We determine the free energy of boundaries at different temperatures from a capillary wave analysis of the thermal fluctuations of the boundaries and find that it varies from $22 \mathrm{meV} / \mathrm{nm}$ at $600 \mathrm{~K}$ to $8 \mathrm{meV} / \mathrm{nm}$ at $650 \mathrm{~K}$. Combining this result with previous measurements of the surface stress difference between the two phases we find that the theory of surface-stress-induced domain formation can quantitatively account for the observed periodicities.
\end{abstract}

DOI: 10.1103/PhysRevB.70.245413

PACS number(s): 81.16.Dn, 68.35.Md, 68.37.Nq, 68.47.De

\section{INTRODUCTION}

When $\mathrm{Pb}$ is deposited on $\mathrm{Cu}(111)$, the surface rapidly rearranges in a remarkable fashion: it self-assembles into patterns composed of domains of a $\mathrm{Pb}$-rich phase and a $\mathrm{Pb}$-poor phase with temperature-dependent periodicities ranging from 40 to $140 \mathrm{~nm} \cdot{ }^{1,2}$ Recently, we have presented evidence that this self-assembly is the result of elastic relaxations at the boundaries between the two phases. ${ }^{3}$ In this paper, we further refine our understanding of the self-assembly by measuring the boundary free energy, one of the factors that are predicted to determine the periodicity.

The phases observed to self-assemble on $\mathrm{Cu}(111)$ are a disordered $\mathrm{Pb} / \mathrm{Cu}$ surface alloy and an incommensurate $\mathrm{Pb}$ overlayer. These atomic structures have been well characterized by low-energy electron diffraction (LEED), ${ }^{4}$ spotprofile-analysis (SPA) LEED, ${ }^{5,6} \mathrm{STM}^{7}$ and surface x-ray diffraction. ${ }^{8}$ The surface alloy phase consists of a random distribution of $\mathrm{Pb}$ atoms substituted in the $\mathrm{Cu}(111)$ surface layer and is the thermodynamically stable phase up to a coverage of $0.22 \mathrm{~Pb}$ atoms per surface $\mathrm{Cu}$ atom. When $\mathrm{Pb}$ is deposited in excess of 0.22 monolayers (ML), the surface dealloys and the $\mathrm{Pb}$ overlayer phase appears. It completely covers the surface at $0.56 \mathrm{ML}$ and has a lattice constant close to $4 / 3$ that of the $\mathrm{Cu}$ substrate. The low-energy boundary between these two phases consists of a surface step: the $\mathrm{Pb}$ overlayer steps down to the alloy phase. A schematic of the striped phase is shown in Fig. 1.

Because the alloy and overlayer phases have different surface stresses, elastic relaxations can occur at the boundary between them. The energetic decrease associated with these relaxations can be large enough to overcome the energetic cost of making the boundaries due to short-ranged atomic interactions - in this case the attractive $\mathrm{Pb}-\mathrm{Pb}$ cohesive interactions which would normally cause overlayer steps to cost energy. To take advantage of these relaxations, periodic arrays of domain boundaries form. The type of domain pattern that forms is predicted (and observed) to depend on the $\mathrm{Pb}$ coverage: when it is such that the two phases have approximately the same areal coverage, a striped domain pattern, as in Fig. 2, is the low-energy structure. On the other hand, when the coverage of one phase is much larger than the other, the equilibrium pattern consists of droplets of one phase inside the other.

At $T=0 \mathrm{~K}$, the repeat distance of the stripe pattern $l_{0}$ is predicted to $b \mathrm{e}^{9-12}$

$$
l_{0}=2 \pi e a \exp \left(\beta / C_{2}\right),
$$

where $\beta$ is the boundary energy per unit length. If the $\mathrm{Cu}$ substrate were elastically isotropic, the parameter $C_{2}$ would be given by

$$
C_{2}=\frac{\Delta \sigma^{2}\left(1-\nu^{2}\right)}{\pi E}
$$

where $\Delta \sigma$ is the stress mismatch, $E$ is Young's modulus, and $\nu$ is Poisson's ratio. ${ }^{10}$ Performing the full anisotropic elasticity calculation for $\mathrm{Cu}$ at $600 \mathrm{~K}$ replaces $\left(1-\nu^{2}\right) / E$ with $0.75 \times 10^{-11} \mathrm{~m}^{2} / \mathrm{N} .{ }^{13}$ The $a$ in Eq. (1) is a short distance

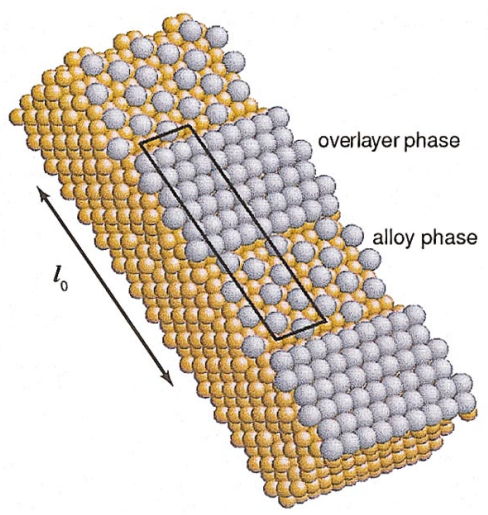

FIG. 1. (Color) A ball model showing the striped phase of $\mathrm{Pb}$ on $\mathrm{Cu}(111)$. The $\mathrm{Pb}$ atoms are grey and the $\mathrm{Cu}$ atoms are gold. 


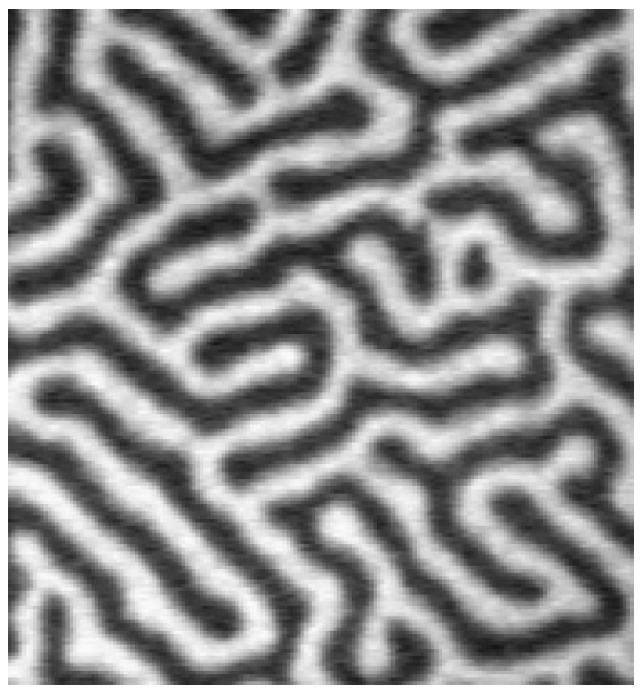

FIG. 2. An example stripe phase observed at $608 \mathrm{~K}$. The $\mathrm{Pb}$ overlayer phase shows up bright in the LEEM images and the $\mathrm{Pb} / \mathrm{Cu}$ surface alloy phase is dark. Note that the stripe phase that has formed is not ideal. As discussed in the text, the length of the stripes limits the wavelengths that we can analyze with the capillary wave analysis. Field of view is $1.7 \mu \mathrm{m}$.

cutoff. The cutoff is necessary because the elastic model leading to Eq. (1) contains no parameter with dimensions of distance, and would be mathematically divergent without one. This difficulty evidently prevents us from predicting $l_{0}$ absolutely. As discussed below it does not, however, stand in the way of predicting observed temperature dependences. Thus, the quasimacroscopic theory leading to Eq. (1) has sufficient physical content to verify the appropriateness of the basic picture-that patterns are formed as a result of competition between boundary energies and stress differences.

The purpose of this paper is to determine how well Eq. (1) accounts for the observed domain boundary periodicities. As previously reported, the stripe periodicity in the $\mathrm{Pb} / \mathrm{Cu}(111)$ system is strongly temperature dependent. ${ }^{2}$ As temperature is increased, a sharp decrease in the feature size is observed, from $140 \mathrm{~nm}$ at $590 \mathrm{~K}$ to $40 \mathrm{~nm}$ at $650 \mathrm{~K}$. To account for this temperature dependence, we assume that the zerotemperature form of Eq. (1) holds, but that $\beta$ should be interpreted as the boundary free energy per unit length and that $\Delta \sigma^{2}$ is the finite-temperature stress mismatch. (That is, we ignore the effects of the thermal disorder in the stripe patterns seen in Fig. 2 on the local stripe width.) In our previous study of this system ${ }^{3}$ we found that the stress mismatch between the two phases was approximately $1.2 \mathrm{~N} / \mathrm{m}$ and did not show a significant temperature dependence. Thus, to explain our observations of a changing domain pattern periodicity we need to assume a decreasing domain boundary free energy with temperature. In this paper we confirm this postulate by presenting measurements of the domain boundary free energy as a function of temperature. We also offer evidence that the source of the temperature dependence is kink excitations in the boundary step edge.

Before proceeding to consider the measurements, it is important to expose a subtle issue raised by the need for a cutoff in the elastic theory underlying Eq. (1). It is that the interpretation of the quantity $\beta$ depends on the value chosen for $a$. If $a$ is taken to be of the order of a few lattice constants, then the value of $\beta$ needed to account for stripe widths will roughly equal the energy needed to break $\mathrm{Pb}-\mathrm{Pb}$ bonds to form a boundary, without elastic relaxation. Thus, $\beta$ would be comparable to a step free energy on $\mathrm{Pb}(111)$. If, however, $a$ is chosen to be large-many lattice constantsthen more of the elastic relaxation energy at the phase boundary would have to be included in $\beta$, in order for Eq. (1) to yield the measured stripe width $l_{0}$.

We deal with this ambiguity by measuring $\beta$ in a way, which, as we will explain below, forces the result to represent the short-ranged $\mathrm{Pb}-\mathrm{Pb}$ interaction, and to include elastic relaxation effects only minimally. Substituting the measured $\beta$ and stripe width into Eq. (1), then produces a value of the cutoff $a$. If the theory is reasonable, the value that emerges should be of the order of a few lattice spacings.

\section{BOUNDARY ENERGY MEASUREMENTS}

To perform a quantitative measurement of the domain boundary energy we used a capillary wave analysis of the thermal fluctuations of steps previously developed to study Si surfaces. ${ }^{14-17}$ Steps on surfaces wander due to the thermal excitations of kinks in the step edge. The thermodynamic consequences of these kink excitations is given by the step stiffness $\widetilde{\beta}$, which is defined as

$$
\widetilde{\beta}(\theta)=\beta(\theta)+\frac{d^{2} \beta}{d \theta^{2}} .
$$

The increase in free energy per unit length when rotating a step by a small angle $\theta$ is $\widetilde{\beta} \theta^{2}$. The stiffness is determined by the kink energy $\epsilon$ : for example, if the density of kinks is sufficiently small, on a (111) surface the stiffness of a closepacked step edge is given by ${ }^{18}$

$$
\widetilde{\beta}=\frac{2 k T}{3 a_{0}} \exp (-\epsilon / k T),
$$

where $a_{0}$ is the nearest-neighbor atomic spacing in the (111) surface and $\epsilon$ is the kink energy. In the capillary wave analysis, $\widetilde{\beta}$ is determined by measuring the amplitude of thermally excited fluctuations on the surface in the following manner. Let $x(y)$ represent the profile of the boundary. If one defines the Fourier components $x_{q}$ by $x(y)=\Sigma_{q} x_{q} e^{i q y}$, then in the absence of any surface stress effects the free energy per unit straight-boundary length of a distorted boundary relative to the straight-boundary energy can be written as ${ }^{14,17}$

$$
f=\frac{1}{2} \sum_{q} q^{2} \widetilde{\beta}\left|x_{q}\right|^{2} .
$$

Equipartition of the energy among the fluctuation modes gives the mean squared amplitude of each mode to be ${ }^{14,17}$ 


$$
A(q)=2\left\langle|x(q)|^{2}\right\rangle=\frac{2 k T}{L \tilde{\beta} q^{2}},
$$

where $L$ is the length of the domain boundary that was analyzed. Thus measurement of $A(q)$ determines $\widetilde{\beta}$.

When the step is also a phase boundary separating phases of different surface stress, there is an additional contribution to the energetics of step fluctuations, caused by elastic relaxation. For example, a straight, isolated phase boundary will become unstable to perturbations with a wavelength above a certain critical value. For sufficiently small wavelengths, we will show that these elastic effects can be neglected and thus the capillary wave analysis measures a stiffness that is determined by short-ranged interactions as in Eq. (4).

Within continuum elasticity theory for an isotropic surface, the free energy of a step is ${ }^{19}$

$$
f=\frac{1}{2} \sum_{q} q^{2}\left[\tilde{\beta}+\left(C_{2} / 2\right) \ln (q a)\right]\left|x_{q}\right|^{2} .
$$

We first note that wave vectors $q a<e^{-2\left(\tilde{\beta} / C_{2}\right)}$ have negative energy; these modes are unstable as mentioned above. Second, we note that the $\ln (a)$ term effectively renormalizes the stiffness $\widetilde{\beta}$. Thus in principle, there is an ambiguity in the definition of the boundary stiffness caused by the choice of the cutoff $a$. However, as before, if $a$ is chosen to be on the order of the lattice constant, one expects the stiffness to be given by something close to Eq. (4), where the kink energy is just determined by atomic cohesive energies. In this case if $q \gg q_{\min }=e^{-2\left(\tilde{\beta} / C_{2}\right)} / a$ one can neglect elastic effects in Eq. (7). Thus if $C_{2}$ is not much greater than $\tilde{\beta}$, there will be a large range of small wavelengths where the energy of distorting the step edge is again given by Eq. (5). In this range of wavelengths, measurement of $A(q)$ provides an estimate of the stiffness due to broken atomic bonds.

Finally, we note that at the temperatures of our experiment, there is no sign of any dependence of the boundary free energy on orientation. For example, the shape of small domains are, to within our experimental resolution, circular. In this situation the stiffness equals the boundary energy $\tilde{\beta}$ $\approx \beta$.

\section{EXPERIMENTAL RESULTS}

Experiments were performed on a $\mathrm{Cu}(111)$ crystal $^{20}$ on which we vapor deposited $\mathrm{Pb}$ from an external evaporation source. Surface structures were imaged with a low-energy electron microscope (LEEM) of Bauer's design. ${ }^{21}$ Details on experimental preparation and the setup have been published elsewhere. ${ }^{22,23}$ Boundary energy measurements were performed at half area fraction, i.e., the amount of $\mathrm{Pb}$ that was deposited was such that the surface alloy and overlayer phase covered half the visible surface area, as in Fig. 2. After the deposition of $\mathrm{Pb}$ and formation of the striped phase, we allowed the domain pattern to stabilize and form a wellequilibrated stripe pattern. A suitable domain boundary was subsequently selected for the analysis. When selecting the domain boundary, the wavelengths that can be analyzed are

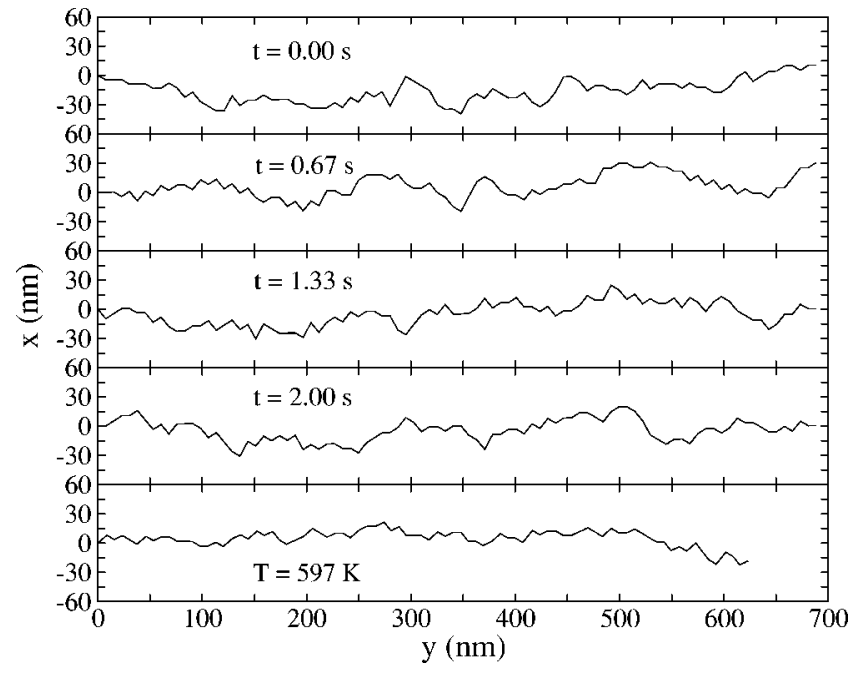

FIG. 3. Measured domain boundary profiles. The four upper panels shows a time sequence of profiles at a temperature of $649 \mathrm{~K}$. The profile in the bottom panel shows the smaller fluctuations at $597 \mathrm{~K}$.

limited by the length of the selected domain boundary, on one hand, and pixelization, on the other. Temporally, we are limited by the video frame rate $(30 \mathrm{~Hz})$, which provides a cutoff for the relaxation times of the capillary waves that we can analyze in the temperature regime where the striped phase forms. Measurements of domain boundary fluctuations were performed from 597 to $649 \mathrm{~K}$. Fluctuations of the domain boundaries were observed to increase with temperature.

The position of the selected domain boundary was then determined using the procedure described by Bartelt and Tromp. ${ }^{16}$ Figure 3 displays several of the domain boundary profiles obtained through this analysis. The domain boundary profile $x(y, t)$ was then decomposed in its Fourier components $x_{q}(t)$. The amplitudes of each of the modes is determined by measuring the time-correlation functions and fitting to an exponential

$$
G_{q}\left(t-t^{\prime}\right)=\left\langle\left|x_{q}(t)-x_{q}\left(t^{\prime}\right)\right|^{2}\right\rangle=A(q)\left(1-e^{-\left[\left(t-t^{\prime}\right) / \tau(q)\right]}\right),
$$

where the characteristic relaxation time of a mode with wave number $q$ is given by $\tau(q)$. The correlation functions obtained from the amplitudes of the Fourier components are shown in Fig. 4. The saturation amplitudes and time constants of the correlation functions decrease with increasing $q$, as expected. The amplitudes of the correlation functions were measured and plotted vs $q^{2}$. An example of this is shown in Fig. 5. Notice that in this figure we only use the amplitudes for small values of $q^{2}$ in our fit. The time constants that we obtain for large $q$ 's are too small to determine them properly, even at video frame rate (30 frames/s). This effect of the fluctuations being too fast to measure is illustrated in Fig. 6 From this plot of $\tau$ vs $q$ we find that the time constants are proportional to $q^{3}$, indicating that the fluctuations are terrace-diffusion limited. ${ }^{24}$ From the same figure we also see that for $q^{3}$ values greater than approximately 0.5 $\times 10^{-3} \mathrm{~nm}^{-3}$ the time constants exceed the frame capture rate. As a result the amplitudes we obtain for $q>0.8 \mathrm{~nm}^{-1}$ 


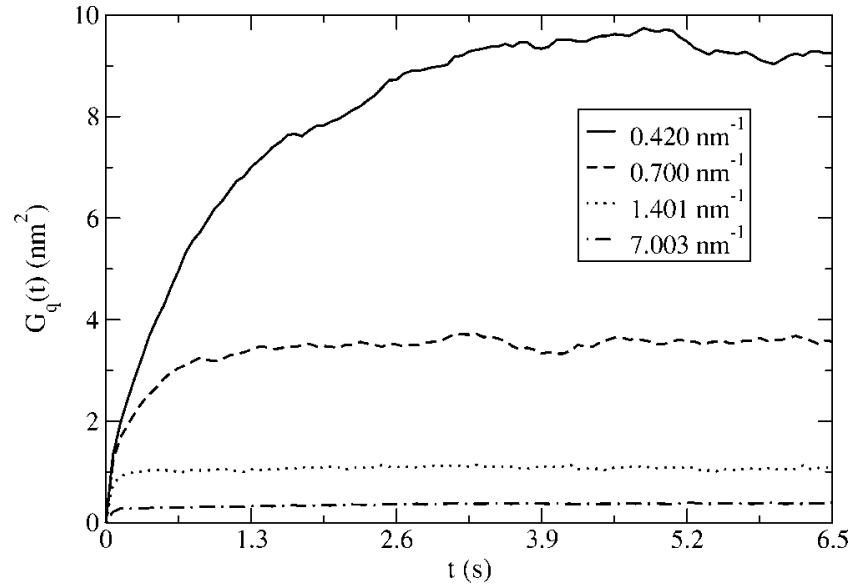

FIG. 4. Correlation functions measured for a fluctuating domain boundary at half coverage at $T=608 \mathrm{~K}$. The plot shows four correlation functions that were obtained for different $q$ values.

are unreliable and cannot be used to obtain a value of the domain boundary stiffness. We repeated the analysis for several different temperatures in the temperature range from 597 to $649 \mathrm{~K}$. The resulting temperature dependence of the domain-boundary energy obtained in this manner is plotted in Fig. 7.

The question now is whether this measured boundary energy, when substituted into Eq. (1) can account for the experimentally observed temperature dependence of stripe periodicity, given a reasonable choice of the cutoff $a$. Figure 8 compares the observed temperature dependence of the stripe width with Eq. (1) making the assumption that $\widetilde{\beta} \approx \beta$. In this comparison $a$ was taken as a fitting parameter with $C_{2}$ $=21 \mathrm{meV} / \mathrm{nm}$, determined by substituting the experimentally estimated value of $\Delta \sigma=1.2 \mathrm{~N} / \mathrm{m}$ into Eq. (2). The value of $a$ that gives the best agreement is indeed reasonable: $3.5 \mathrm{~nm}$, roughly twelve $\mathrm{Pb}-\mathrm{Pb}$ atomic spacings, or four unit cells of the overlayer phase. This value of $a$ would imply that continuum elasticity theory fails to describe elastic distortions within $3.5 \mathrm{~nm}$ of the step edge. With this choice of $a$, the

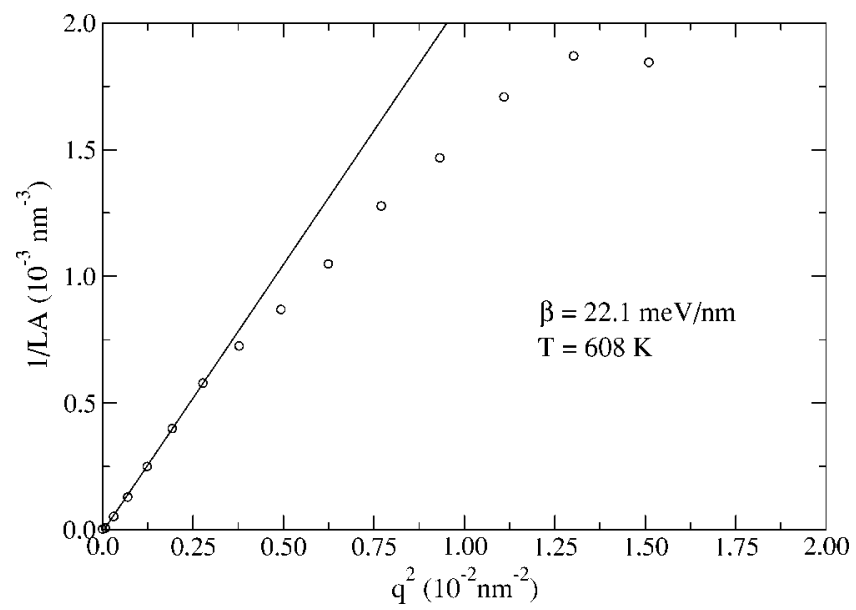

FIG. 5. Measured amplitudes as a function of $q^{2}$ at $T=608 \mathrm{~K}$. From the measurement shown in this figure we obtain a boundary energy value of $22.1 \mathrm{meV} / \mathrm{nm}$.

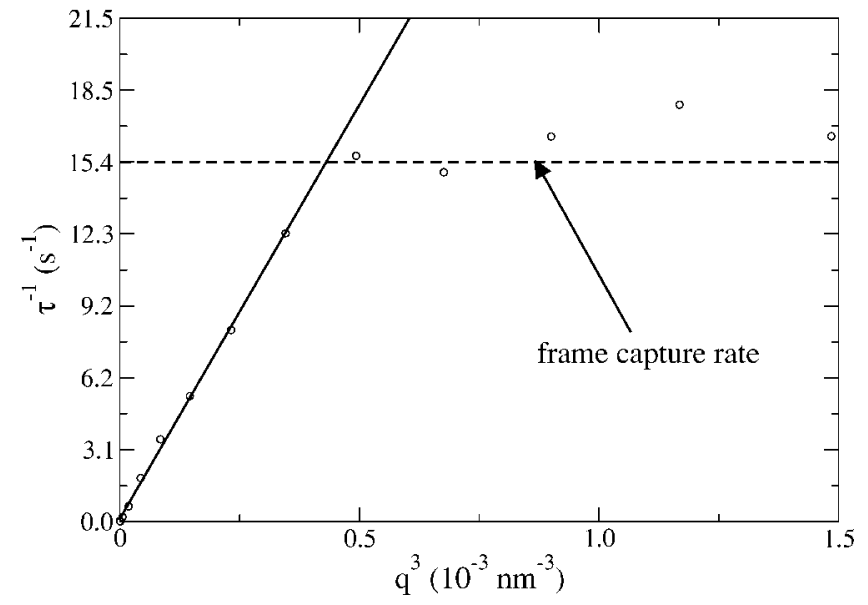

FIG. 6. Estimated relaxation times as a function of $q^{3}$ at $T$ $=608 \mathrm{~K}$. The dashed line indicates the rate at which frames from the LEEM movies were digitized.

agreement between Eq. (1) and experiment is very good.

Given this estimate of the $a$ associated with our values of $\widetilde{\beta}$, we can check the validity of neglecting the elastic effects of Eq. (7) in the capillary wave analysis. At $608 \mathrm{~K}, q_{\min }$ $=\exp \left(-4 \widetilde{\beta} / C_{2}\right) / a \approx 5 \times 10^{-3} \mathrm{~nm}^{-1}$. The $q$ values used in the fits of Fig. 5 were greater than $10^{-2} \mathrm{~nm}^{-1}$, and thus in a regime where the capillary analysis is applicable.

\section{THE ENERGETICS OF BOUNDARY FLUCTUATIONS: COMPARISON WITH FIRST-PRINCIPLES CALCULATIONS}

We address next the source of the thermal excitations in the step edge which gives rise to the temperature dependence of the boundary free energy. In particular, is the temperature dependence consistent with that expected for kink excitations in the step edge, or does one have to invoke more complicated effects such as a temperature dependence of segregation to the step edge? To help answer this question, we first interpret the measured boundary free energies by making the crude assumption that the energetic cost of the step edge is

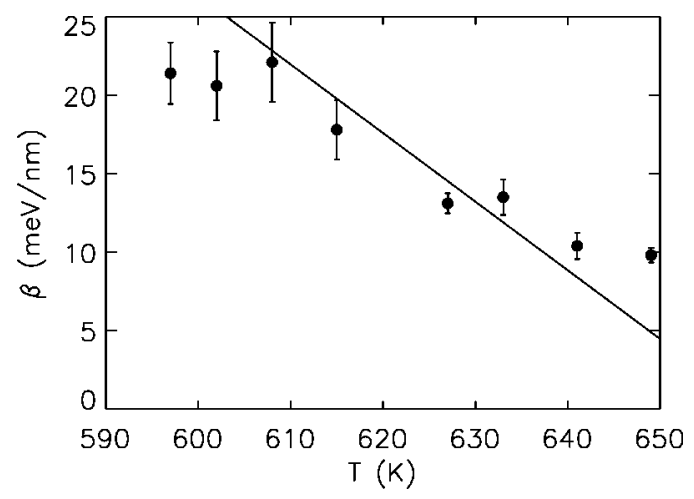

FIG. 7. The solid circles show the temperature dependence of the boundary free energy obtained through the capillary wave analysis. The solid line shows the single-parameter fit to the Ising model discussed in the text [Eqs. (9)-(11)]. 


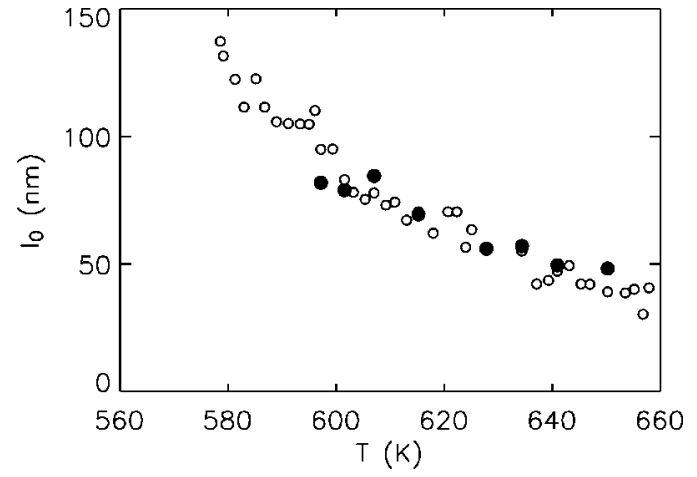

FIG. 8. The characteristic feature size (stripe width) as a function of temperature. Open circles are data points obtained directly from the LEEM images. Filled circles were obtained indirectly through the separate measurements of $\beta$ and $\Delta \sigma$ and Eq. (1). The value of $a$ that was used to reproduce the feature size from our measurements of $\beta$ and $\Delta \sigma$ was $3.5 \mathrm{~nm}$.

due to broken nearest-neighbor $\mathrm{Pb}-\mathrm{Pb}$ bonds. In this case the temperature dependence of the step free energy due to thermal kink excitations in the step edge is known from the exact solution of the triangular Ising model. ${ }^{25}$ The observed average orientation of the boundaries ${ }^{13}$ is $30^{\circ}$ from the close packed step edge direction. For this orientation, the temperature dependence of the step energy is given by

$$
\widetilde{\beta}=\frac{2 k T}{\sqrt{3}} \cosh ^{-1}[(A-1) / 2],
$$

with

$$
A=\frac{2 x}{(1-x)^{2}}+\frac{(1-x)^{2}}{2 x}+1
$$

and

$$
x=\tanh \left[\beta_{C P}(0) / 4 k T\right],
$$

where $\beta_{C P}(0)$ is the zero temperature close-packed step energy. At high temperature $\beta(T)$ decreases linearly with $T$. Figure 7 superimposes a least squares fit of the Ising model result (9) to the experimentally observed step free energies. This single parameter fit yielded a value of $154 \mathrm{meV} / \mathrm{nm}$ for $\beta_{C P}(0)$. The difference between the close packed and $30^{\circ}$ rotated free energies are insignificant at the experimental temperatures: the computed ratio of this step free energy to that of the close-packed direction is less than $1.0004,{ }^{13}$ consistent with the observed circular islands and the assumption that $\beta \approx \widetilde{\beta}$.

To determine if the deduced $154 \mathrm{meV} / \mathrm{nm}$ is a reasonable boundary energy, and thus to check that the simple kink excitation picture of the temperature dependence is plausible, we have used density functional theory to compute the boundary energy. We first optimized the geometry of a periodic model $\mathrm{Pb}$ overlayer in a $4 \times 4 \sqrt{3}$ unit cell. On a six layer, $\mathrm{Cu}(111)$ slab, with $32 \mathrm{Cu}$ atoms per layer, we placed a $\mathrm{Pb}$ layer containing $18 \mathrm{~Pb}$ atoms. Ab initio geometric relaxation of this covered slab yielded a total energy $E_{\text {overlayer }}$. We next computed the energy of a six-layer $\mathrm{Cu}(111)$ slab, in which seven of the top layer $\mathrm{Cu}$ atoms were replaced by $\mathrm{Pb}$ atoms. The optimized energy in this case $E_{\text {alloy }}$ represents that of a surface alloy phase with a surface $\mathrm{Pb}$ concentration of $7 / 32(\approx 0.22)$ ML. Finally, we optimized a $4 \times 8 \sqrt{3}$, sixlayer $\mathrm{Cu}(111)$ slab whose top layer had $18 \mathrm{~Pb}$ overlayer atoms adsorbed over half of it and seven substitutional $\mathrm{Pb}$ atoms located similarly to the pure alloy slab's, a configuration shown in Fig. 1. The energy in this case $E_{\text {striped }}$, is less than $E_{\text {overlayer }}+E_{\text {alloy }}$ by twice the average boundary energy, twice because each overlayer stripe is defined by two steps, one on each side.

The calculations were done using the VASP implementation ${ }^{26-29}$ of density functional theory ${ }^{30,31}$ and the PW91 GGA version of exchange and correlation..$^{32,33}$ VASP represents electron-nucleus interactions in terms of ultrasoft pseudopotentials, ${ }^{34-37}$ minimizing the size of the plane-wave basis set needed for convergence. We used a plane-wave cutoff of $234 \mathrm{eV}$. Geometry optimizations were done with the lower three $\mathrm{Cu}$ layers of each slab held fixed at bulk $\mathrm{Cu}$ relative positions with a GGA value of the $\mathrm{Cu}-\mathrm{Cu}$ spacing $=0.2575 \mathrm{~nm}$, compared to the experimental number 0.2553 $\mathrm{nm}$. The calculations in $4 \times 4 \sqrt{3}$ unit cells were performed using a $2 \times 2$ sample of the surface Brillouin zone. For consistency, the $4 \times 8 \sqrt{3}$ calculation was done using a $2 \times 1$ sample. The Neugebauer-Scheffler method, as implemented by Kresse, ${ }^{38,39}$ was used to compensate for the unphysical contact-potential difference between the slab surfaces that exists because $\mathrm{Pb}$ atoms were only adsorbed on the upper surface. The Methfessel-Paxton Fermi-Level smearing method (width $=0.2 \mathrm{eV}$ ) was used to accelerate electronic relaxation. ${ }^{40}$

The result of the comparison of slab energies is an estimated domain boundary energy of $240 \mathrm{meV} / \mathrm{nm}$. This compares with the experimental value of $154 \mathrm{meV} / \mathrm{nm}$. Given the crude assumptions of the nearest-neighbor Ising model and the fact that we do not know the actual step edge morphology, specifically, where the nearest $\mathrm{Pb}$ atoms lie relative to an overlayer boundary, this quite good level of agreement shows that it is plausible that kink excitations are responsible for the observed temperature dependence of the boundary energy. Note that in earlier work concerning $\mathrm{Pb}$ stripe islands on $\mathrm{Pb}(111)$, the similarly computed boundary energy ${ }^{18}$ was $88 \mathrm{meV} / \mathrm{atom}$, or $246 \mathrm{meV} / \mathrm{nm}$. The similarity of these values seems no accident. It reflects the fact that the main source of the boundary energy is the same in both cases, namely the broken $\mathrm{Pb}-\mathrm{Pb}$ bonds that define an overlayer stripe's boundaries. The fact that there is $\mathrm{Cu}$ beneath the stripe in one case and $\mathrm{Pb}$ in the other makes little difference. Thus it seems that thermally broken $\mathrm{Pb}-\mathrm{Pb}$ bonds are most likely responsible for the large temperature dependence of the stripe periodicity.

\section{CONCLUSIONS}

We have shown that the changing periodicity of selfassembled domain patterns in the $\mathrm{Pb} / \mathrm{Cu}(111)$ system can be attributed to a changing domain boundary free energy. It, in turn, is due to increased kink excitations in the step that separates the domains. 
In previous work ${ }^{3}$ we found that the magnitude of the stress mismatch between the $\mathrm{Pb}$ phases which causes the self-assembly of $\mathrm{Pb} / \mathrm{Cu}$ is not anomalous. In this paper, we have shown that the boundary free energy which determines the periodicity of the domain patterns also has a typical value. Taken together these results show that the driving forces for the dramatic self-assembly observed in the $\mathrm{Pb} / \mathrm{Cu}$ system are not the result of a special energetic situation. Another interesting feature of the $\mathrm{Pb} / \mathrm{Cu}$ system is the rapid mass transport which occurs during the equilibration of the domain patterns. ${ }^{2}$ We are now investigating whether the atomic mechanisms that lead to this equilibration are unique to $\mathrm{Pb} / \mathrm{Cu}$.

\section{ACKNOWLEDGMENTS}

This work was supported by the Department of Energy, Office of Basic Energy Sciences, Division of Materials Sciences and Engineering and was performed at Sandia National Laboratories, a multiprogram laboratory operated by Sandia Corporation, a Lockheed Martin company, for the United States Department of Energy under Contract no DEAC04-94AL85000.
${ }^{1}$ R. Plass, J. A. Last, N. C. Bartelt, and G. L. Kellogg, Nature (London) 412, 875 (2001).

${ }^{2}$ R. Plass, N. C. Bartelt, and G. L. Kellogg, J. Phys.: Condens. Matter 14, 4227 (2002).

${ }^{3}$ R. van Gastel, R. Plass, N. C. Bartelt, and G. L. Kellogg, Phys. Rev. Lett. 91, 055503 (2003).

${ }^{4}$ J. Henrion and G. E. Rhead, Surf. Sci. 29, 20 (1972).

${ }^{5}$ G. Meyer, M. Michailov, and M. Henzler, Surf. Sci. 202, 125 (1988).

${ }^{6}$ B. H. Müller, Th. Schmidt, and M. Henzler, Surf. Sci. 376, 123 (1997).

${ }^{7}$ C. Nagl O. Haller, E. Platzgummer, M. Schmid, and P. Varga, Surf. Sci. 321, 237 (1994).

${ }^{8}$ Y. S. Chu, I. K. Robinson, and A. A. Gewirth, Phys. Rev. B 55, 7945 (1997).

${ }^{9}$ V. I. Marchenko, JETP Lett. 33, 381 (1981).

${ }^{10}$ O. L. Alerhand, D. Vanderbilt, R. D. Meade, and J. D. Joannopoulos, Phys. Rev. Lett. 61, 1973 (1988).

${ }^{11}$ D. Vanderbilt, Surf. Sci. Lett. 268, L300 (1992).

${ }^{12}$ K.-O. Ng and D. Vanderbilt, Phys. Rev. B 52, 2177 (1995).

${ }^{13}$ F. Léonard, N. C. Bartelt, and G. L. Kellogg, (unpublished).

${ }^{14}$ N. C. Bartelt, J. L. Goldberg, T. L. Einstein, E. D. Williams, J. C. Heyraud, and J. J. Metois, Phys. Rev. B 48, 15453 (1993).

${ }^{15}$ N. C. Bartelt, R. M. Tromp, and E. D. Williams, Phys. Rev. Lett. 73, 1656 (1994).

${ }^{16}$ N. C. Bartelt and R. M. Tromp, Phys. Rev. B 54, 11731 (1996).

${ }^{17}$ H. C. Jeong and E. D. Williams, Surf. Sci. Rep. 34, 175 (1999).

${ }^{18}$ P. J. Feibelman, Phys. Rev. B 62, 17020 (2000); 65, 129902(E) (2002)

${ }^{19}$ F. Léonard and J. Tersoff, Appl. Phys. Lett. 83, 72 (2003).
${ }^{20}$ R. J. I. M. Koper, http://www.surface-prep-lab.com/

${ }^{21}$ E. Bauer, Rep. Prog. Phys. 57, 895 (1994).

${ }^{22}$ R. A. Plass and G. L. Kellogg, Surf. Sci. 470, 106 (2000).

${ }^{23}$ G. L. Kellogg and R. Plass, Surf. Sci. 465, L777 (2000).

${ }^{24}$ N. C. Bartelt, T. L. Einstein, and E. D. Williams, Surf. Sci. 312, 411 (1994).

${ }^{25}$ V. A. Shneidman and R. K. P. Zia, Phys. Rev. B 63, 085410 (2001).

${ }^{26}$ G. Kresse and J. Hafner, Phys. Rev. B 47, 558 (1993).

${ }^{27}$ G. Kresse and J. Hafner, Phys. Rev. B 49, 14251 (1994).

${ }^{28}$ G. Kresse and J. Furthmüller, Comput. Mater. Sci. 6, 15 (1996).

${ }^{29}$ G. Kresse and J. Furthmüller, Phys. Rev. B 54, 11169 (1996).

${ }^{30}$ P. Hohenberg and W. Kohn, Phys. Rev. 136, B864 (1964).

${ }^{31}$ W. Kohn and L. J. Sham, Phys. Rev. 140, A1133 (1965).

${ }^{32}$ J. P. Perdew, in Electronic Structure of Solids '91, edited by P. Ziesche and H. Eschrig (Akademie Verlag, Berlin, 1991).

${ }^{33}$ J. P. Perdew, J. A. Chevary, S. H. Vosko, K. A. Jackson, M. R. Pederson, D. J. Singh, and C. Fiolhais, Phys. Rev. B 46, 6671 (1992).

${ }^{34}$ D. Vanderbilt, Phys. Rev. B 41, 7892 (1990).

${ }^{35}$ A. Pasquarello, K. Laasonen, R. Car, C. Lee, and D. Vanderbilt, Phys. Rev. Lett. 69, 1982 (1992).

${ }^{36}$ K. Laasonen, A. Pasquarello, R. Car, C. Lee, and D. Vanderbilt, Phys. Rev. B 47, 10142 (1993).

${ }^{37}$ G. Kresse and J. Hafner, J. Phys.: Condens. Matter 6, 8245 (1994).

${ }^{38}$ J. Neugebauer and M. Scheffler, Phys. Rev. B 46, 16067 (1992).

${ }^{39}$ G. Kresse, VASP Guide, http://cms.mpi.univie.ac.at/VASP/guide/ node $143 . \mathrm{html}$

${ }^{40}$ M. Methfessel and A. T. Paxton, Phys. Rev. B 40, 3616 (1989). 